\title{
COMPETENCE TO FORM ELECTRICAL CONNECTIONS IS RESTRICTED TO GROWING NEURITES IN THE SNAIL, HELISOMA ${ }^{1}$
}

\author{
ROBERT D. HADI.EY ${ }^{2}$ AND S. B. KATER ${ }^{3}$ \\ Department of Zoology, University of Iowa, Iowa City, Iowa 52242
}

Received September 28, 1982; Revised December 20, 1982; Accepted December 22, 1982

\begin{abstract}
Identified neurons of the snail, Helisoma, undergo extensive remodeling in response to axotomy, including the formation of specific sets of novel electrical connections. This communication addresses the question of why, under the conditions employed, some neurons readily form new connections with a single "test" neuron, whereas others do not.

The present experiments are a test of the hypothesis that, for these adult neurons, competence to form electrical connections is restricted to pairs of neurons with interacting regions of active outgrowth. Morphological observations demonstrated profuse overlapping outgrowth from neurons which formed electrical connections, whereas neurons which did not connect displayed no simultaneous new outgrowth, although there could be regions of physical overlap or proximity. The causal relationship between growth and the ability to form new connections was tested more directly by two means: (1) Previously nonconnecting neurons were recruited into the connectivity pattern by axotomy-induced growth. (2) Previously connecting neurons did not connect when they were not induced to grow. Thus, growth or lack of growth is an effective discriminator for determining specific sets of interconnected neurons.
\end{abstract}

Recent work from our laboratory has shown a significant potential for extensive remodeling of connections between identified neurons of the snail, Helisoma. Axotomy-evoked neuroplastic responses include restoration of broken connections (Murphy and Kater, 1978, 1980), changes in the efficacy of chemical and electrical connections (Bulloch et al., 1980; Murphy et al., 1983), and the formation of novel electrical synapses between previously unconnected neurons (Bulloch and Kater, 1981, 1982). This major reorganization involves every neuron thus far examined in the buccal ganglion and, on initial inspection, seems chaotic.

When viewed from the perspective of a single identified neuron, a highly stereotyped and predictable set of changes becomes apparent. Many new connections are made, but only some are stabilized. For instance, neuron

\footnotetext{
1 This work was suppored by Public Health Service Grants NS 15350, NS 18819 and AM 19858. We wish to thank Drs. C. Cohan, J. Denburg, J. Frankel, R. Wong, and C. -F. Wu, as well as Joanne Kater, for their valuable comments on the manuscript and Paul Gade for his expert technical and photographic assistance. We also thank the anonymous journal reviewers for their helpful and illuminating comments on the manuscript.

${ }^{2}$ Present address: Department of Physiology and Biophysics, Wash ington University Medical School, 660 S. Euclid Avenue, St. Louis, MO 63110.

${ }^{3}$ To whom correspondence should be addressed.
}

5 forms a highly specific set of novel connections, but, of these, only the connection between neurons $5 \mathrm{R}$ and $5 \mathrm{~L}$ is stable. This reorganization is an example, for identified neurons, of a process of selective synaptic elimination and stabilization (e.g., Redfern, 1970; Dennis and Yip, 1978; Purves and Lichtman, 1980).

The present study asks about an even more stringent level of selectivity. Why are some neurons (e.g., neuron 19 and the other protractor motoneurons) excluded from ever connecting with neuron 5 (Bulloch and Kater, 1982), even though physical proximity is ensured by neuron 5's axotomy-induced growth? A priori, one could consider two possible explanations for the exclusion of any set of neurons from connecting with neuron 5 . (I) The neurons could be inherently and permanently incompetent to connect with neuron 5; or (2) this set of neurons may have existed in a particular state or condition which did not allow them to establish new connections.

This communication shows that the behavior of this set of excluded neurons is not inherently determined. Rather, when these neurons are shifted from a stable morphology to a growing state, they connect with neuron 5 and behave like many other neurons in neuron 5's remodeled connectivity pattern. These experiments are the initial test of our working hypothesis that the first step in the establishment of electical connections in this system is the interaction between actively growing regions of potential partner cells. 'I'hese initial connections 
can only be formed by growing neurons, after which selective mechanisms can act to further refine a final neuronal circuit.

\section{Materials and Methods}

Dissection and media. Stocks of Helisoma trivolvis were maintained and prepared for sterile dissection as previously described (Wong et al., 1981; Hadley et al., 1982). After dissection to expose the CNS (Kater and Kaneko, 1972), buccal ganglia were excised by cutting all nerve roots at their distal extremes and were incubated for $15 \mathrm{~min}$ in antibiotic saline. Neurons were axotomized by crushing esophageal nerve trunks (ETs) or the commissure between the paired buccal ganglia with jeweler's forceps (Fig. 1). The site of axotomy has previously been demonstrated to be an important determinant of neuronal growth patterns. That is, distal axotomy resulted in predominantly peripheral sprouting, whereas axotomy near the cell body produced extensive central sprouting (Murphy and Kater, 1980; Bulloch and Kater, 1982; Hadley et al., 1982). For some experiments (Figs. 9 and 10) buccal ganglia were cultured with portions of esophageal tissue (neuron 5 target tissue) left attached to the distal ends of the ETs to provide conditions conducive to neuron 5 morphological stability (Cohan et al., 1983). These preparations were cultured in HL-15 medium (see below).

Helisoma normal saline consisted of (in $\mathrm{mm}$ ): $\mathrm{NaCl}$, 51.3; KCl, $1.7 ; \mathrm{CaCl}_{2}, 4.1 ; \mathrm{MgCl}_{2}, 1.5$; HEPES buffer, 5 at $\mathrm{pH}$ 7.3. Sterile antibiotic saline was prepared by adding $500 \mathrm{units} / \mathrm{ml}$ of penicillin, $0.5 \mathrm{mg} / \mathrm{ml}$ of streptomycin, and $1.25 \mu \mathrm{g} / \mathrm{ml}$ of Fungizone to autoclaved normal saline. Culture was either in hanging drops of Helisoma blood (see below) or half-strength Liebowitz L-15 medium with Helisoma salts and co-cultured Helisoma brains (HL-15, 3 brains $/ \mathrm{ml}$; see Wong et al., 1981 for details). Cultures were incubated in air at 22 to $25^{\circ} \mathrm{C}$ in a humidified cabinet.

Blood cultures. Blood was collected for use as a culture medium by first placing large adult "donor" snails (18 to $20 \mathrm{~mm})$ in clean artificial pond water $(1 \mathrm{gm} / \mathrm{gallon}$ of Instant Ocean) for $15 \mathrm{~min}$. Shells were carefully cut back

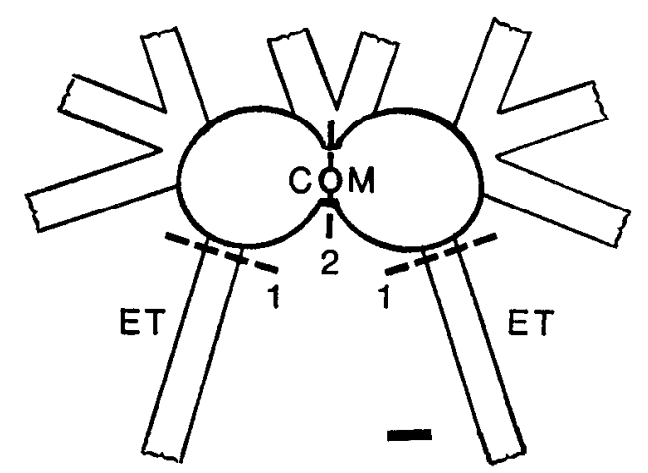

Figure 1. Schematic representation of the preparation employed for these experiments. Buccal ganglia are removed from the animal by culting all nerve trunks peripherally. "Proximal axotomy" is achieved by crushing the esophageal nerve trunks (position 1, dashed lines) or the commissure between the paired ganglia (position 2, dashed line). Relative lengths of nerve trunks are shown approximately to scale. Scale bar, $100 \mu \mathrm{m}$. to the level of the foot mass, and the animals were placed in a $25 \%$ Listerine solution in normal saline for $20 \mathrm{~min}$ then were allowed to recover in sterile antibiotic saline for another $20 \mathrm{~min}$. Shells were then thoroughly dried with sterile cotton gauze and cut back to behind the level of the foot, to the mantle margin. Mucus was removed from the exposed animal by gently wiping with sterile gauze. Animals were bled by first prodding the anterior part of the foot to induce withdrawal of the animal; this increased hemocoel pressures so that blood was readily expelled when the posterior-dorsal part of the foot was lanced with sharp sterile forceps. Blood was collected in sterile, nonheparinized hematocrit tubes as it flowed down to the bottom of the cleaned shell. Tubes were plugged and centrifuged for $3 \mathrm{~min}$ to remove any cells or debris. Refrigerated blood could be used for up to 1 week, although most was used fresh. Donor snails yielded an average of about $80 \mu \mathrm{l}$ of blood each.

Hanging drop cultures were in single, 15 - to $20-\mu$ l drops of blood in $35-\mathrm{mm}$ plastic culture dishes (Falcon no. 1008), inverted onto their lids. With moistened sterile gauze on the floor of the chamber, there was no apparent loss in volume due to evaporation for at least 1 week. For culture durations longer than 3 days, ganglia were transferred to fresh blood at 3-day intervals. Although no antibiotics were added to the blood, contamination was negligible.

Electrophysiology and dye injection. For electrophysiological measurements, ganglia were removed from culture and stabilized under drops of an agar/gelatin mixture (Hadley et al., 1982). Measurements were performed by conventional means. Fiber-filled micropipettes filled with $4 \mathrm{M}$ potassium acetate (resistances $=20$ to 40 megohms) were connected to a unity gain, high impedance amplifier. Signals were displayed on a Tektronix 502 oscilloscope and a Brush 220 chart recorder. Neurons were penetrated under a dissecting stereomicroscope in normal saline.

Electrical coupling was measured by intracellular microelectrode recording and hyperpolarizing current injection via a balanced bridge circuit into one of the cell pair. Coupling coefficients were determined as the ratio of postsynaptic to presynaptic voltage deflection on the oscilloscope face (Bennett, 1966). Coupling measurements were made in low $\mathrm{Ca}$, high $\mathrm{Mg}(0 \mathrm{~mm} \mathrm{Ca}, 15 \mathrm{~mm}$ $\mathrm{Mg}$ ) saline which blocked ongoing chemical inputs whose conductance shunts could often obscure electrical coupling with coefficients less than 0.05 to 0.10 . In low $\mathrm{Ca}$, high $\mathrm{Mg}$ saline, coupling coefficients as low as 0.01 could be accurately measured. The lower limit of detection was estimated to be about 0.0025 . Coupling between 0.0025 and 0.01 could not be accurately measured and was assigned a value of 0.005 for computation of mean coupling. Only pairs of cells with undetectable coupling $(<0.0025)$ were scored as lacking a connection. Coupling coefficients between pairs of cells often were not symmetrical. When the input resistances of the cells were unequal, coupling measured from the lower to the higher resistance cell was higher than in the reverse direction. In these cases, the higher value for the coupling coefficient was always chosen for computation. Measurements of neuron 5-neuron 19 coupling were made on ipsilateral cell pairs only. Neuron 4-neuron 4 coupling increased 
with axotomy and culture (Murphy et al., 1983) to such a degree that 5-4 coupling with ipsilateral or contralateral pairs was essentially identical. Comparisons of coupling data for different experimental treatments were made using the Student's $t$ test for significance and an unmatched variance correction (Dixon and Massey, 1969). All sample group values are reported as mean \pm SEM.

The morphology of neurons was examined by staining them with Lucifer Yellow CH (Stewart, 1978) injected intracellularly by iontophoresis. Lucifer Yellow (3\% in distilled water) was injected by DC hyperpolarizing currents $10 \mathrm{nA}$ in amplitude and $2 \mathrm{~min}$ in duration. Specimens were fixed overnight in phosphate-buffered $4 \%$ formaldehyde $(\mathrm{pH} 7.4)$ at $4{ }^{\circ} \mathrm{C}$, dehydrated through an alcohol series, cleared in methyl salicylate, and mounted in clearing agent on microscope slides. Viewing and photography were through a Zeiss Universal microscope with epi-illumination.

\section{Results}

Helisoma buccal ganglion neurons. The normal morphologies of buccal neurons 5, 4, and 19 (nomenclature of Kater and Rowell, 1973) are illustrated in Figure 2. Each neuron is one of a mirror image pair (e.g., $5 \mathrm{R}$ and $5 \mathrm{~L}, 4 \mathrm{R}$ and $4 \mathrm{~L}$, etc.). The normal electrotonic connections in acutely dissected ganglia are as stereotyped as the neuronal morphologies (Fig. 2). In general, all neurons are connected to their contralateral counterparts via an axon in the commissure (e.g., $4 \mathrm{R}$ and $4 \mathrm{~L}, 19 \mathrm{R}$ and $19 \mathrm{~L}$ ). Neurons 5 are an exception; they are monopolar with an axon only in the ET and are not normally connected to one another. Other connections which are absent in acutely dissected ganglia are 5-4, 19-5, and 19-4. This qualitative pattern of normal coupling is rigidly adhered to. Quantitatively, coupling coefficients between pairs of neurons also fall within a narrow range. For instance, coupling between neurons $4 \mathrm{R}$ and $4 \mathrm{~L}$ is $0.54 \pm 0.03(n=$ 13; Murphy et al., 1983). The morphological and physiological predictability of this system allow high resolution examination of changes in status following axotomy.

Although the connections described above are stable in normal animals, novel connections between previously unconnected neurons (e.g., 5-5 and 5-4, * in Fig. 2) form subsequent to ET crush (Bulloch and Kater, 1981), whereas under these same conditions 5-19 (**) connections were never detected. In this paper we ask why a set of buccal neurons (including neuron 19) never connected with neuron 5 . We assess below the competence of neurons to form electrotonic connections under two alternative conditions: (1) when one of the neuron pair had stable central morphology and was not growing; or (2) when both neurons were generating central, growing neurites. Central growth is a function of the proximity of axotomy to the cell body (Murphy and Kater, 1978; Bulloch and Kater, 1982; Hadley et al., 1982) and thus could be evoked reliably in particular neurons by proximal crushes of selected nerves. This central growth was examined as a possible substrate for formation of novel connections.

Conditions for the study of novel connections. Experimentally advantageous quantitative differences were found when blood culture was compared with in vivo
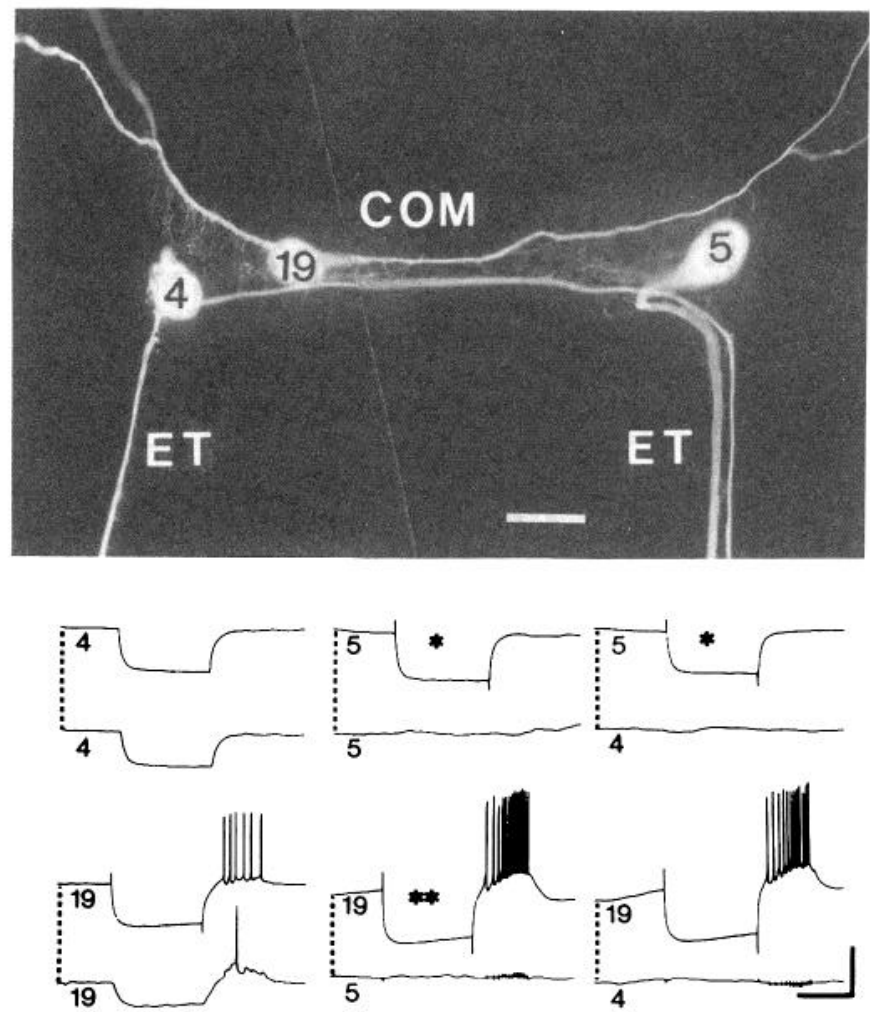

Figure 2. Normal buccal ganglion neuronal morphology and electrical connections. Neuron 5 is monopolar and extends out only the ipsilateral esophageal nerve trunk $(E T)$, whereas neuron 4 is bipolar and extends across the buccal commissure $(C O M)$ and out the contralateral ET as well. Neuron 19 is bipolar, has no axons in the ETs, and extends across the commissure and out ipsilateral and contralateral ventrobuccal and heterobuccal nerves. Each of the Lucifer Yellow-injected neurons shown here is one of a bilaterally symmetrical pair, but, for clarity, only one of each pair was injected. These morphologies are seen in virtually every injected neuron 4,5 , or 19. In acutely dissected buccal ganglia, neurons 4 and 19 are connected to their contralateral mates by electrical synapses (4-4 and 19-19 records), whereas the monopolar neuron 5 is not (5-5 record). No connections are seen for 5-4, 19-5, or 19-4 pairs. Current was injected into neurons in upper traces. Calibrations: $100-\mu \mathrm{m}$ bar in photograph. Electrical records: all top traces, 20 $\mathrm{mV}$; bottom traces of 4-4 and 19-19, $10 \mathrm{mV}$; others, $5 \mathrm{mV} ; 2 \mathrm{sec}$ for all.

(Murphy and Kater, 1980) or in vitro culture in HL-15 medium (Hadley et al., 1982). In all cultures, novel 5-5 connections reliably formed, and no significant differences in strength were detected among the three culture conditions (Table I). In contrast, 5-4 peak coupling coefficients were 3 - to 20 -fold higher in blood-cultured ganglia. While values of coupling coefficients were greater in blood culture, the time courses of establishment and elimination of connections were similar among culture conditions (cf. Fig. 3 and Bulloch and Kater, 1982). The time course of 5-5 and 5-4 coupling strengths in blood culture lagged behind that of in vivo culture by about 1 day, and elimination of 5-4 coupling was not yet completed for most preparations by day 7 ( 6 of 10 preparations remained weakly coupled; range $=0.005$ to 0.10 ) .

These data on 5-5 and 5-4 coupling were obtained as a 
composite from single measurements in more than 50 different ganglia. Similar results were seen in two ganglia that were serially assayed on days 2 and $4 ; 5-5$ strengths rose whereas 5-4 strengths declined, in accord with the composite time course. Because blood culture provided the most favorable conditions for formation of these connections, we used it to assess whether additional neurons could be induced to connect to neuron 5 .

Growth and novel connections. Our first indication of the role of growth in determining connectivity patterns after ET crush came from morphological observations on two distinct classes of neurons: those which couple to neuron 5 and those which do not. Central growing neurites were apparent only within the group of included neurons and not within the group of excluded neurons.

In buccal ganglia which were cultured with bilaterally

TABLE I

Peak 5-5 and 5-4 coupling coefficients in different culture media

\begin{tabular}{lccc}
\hline & In Vivo Culture $^{\alpha}$ & HL-15 Culture $^{b}$ & $\begin{array}{c}\text { Helisoma } \text { Blood } \\
\text { Culture }\end{array}$ \\
\hline Peak 5-5 $^{c}$ & $0.21 \pm 0.06$ & $0.17 \pm 0.03$ & $0.23 \pm 0.03$ \\
& $(8)$ & $(9)$ & $(8)$ \\
Peak 5-4 $^{d}$ & $0.07 \pm 0.03$ & $0.01 \pm 0.01$ & $0.24 \pm 0.03^{*}$ \\
& $(12)$ & $(9)$ & $(11)$ \\
\hline
\end{tabular}

${ }^{a}$ From Bulloch and Kater (1981).

${ }^{b}$ From Hadley et al. (1982).

${ }^{c}$ Values on day 5 of culture. All values are mean $\pm \operatorname{SEM}(n)$. No significant differences between the values were detected $(t$ test).

${ }^{d}$ Values on days 1,2 , and 2 for in vivo, HL-15, and blood, respectively. Blood $5-4$ values $(*)$ are significantly greater than values in vivo or in HL-15 ( $p<0.001, t$ test).
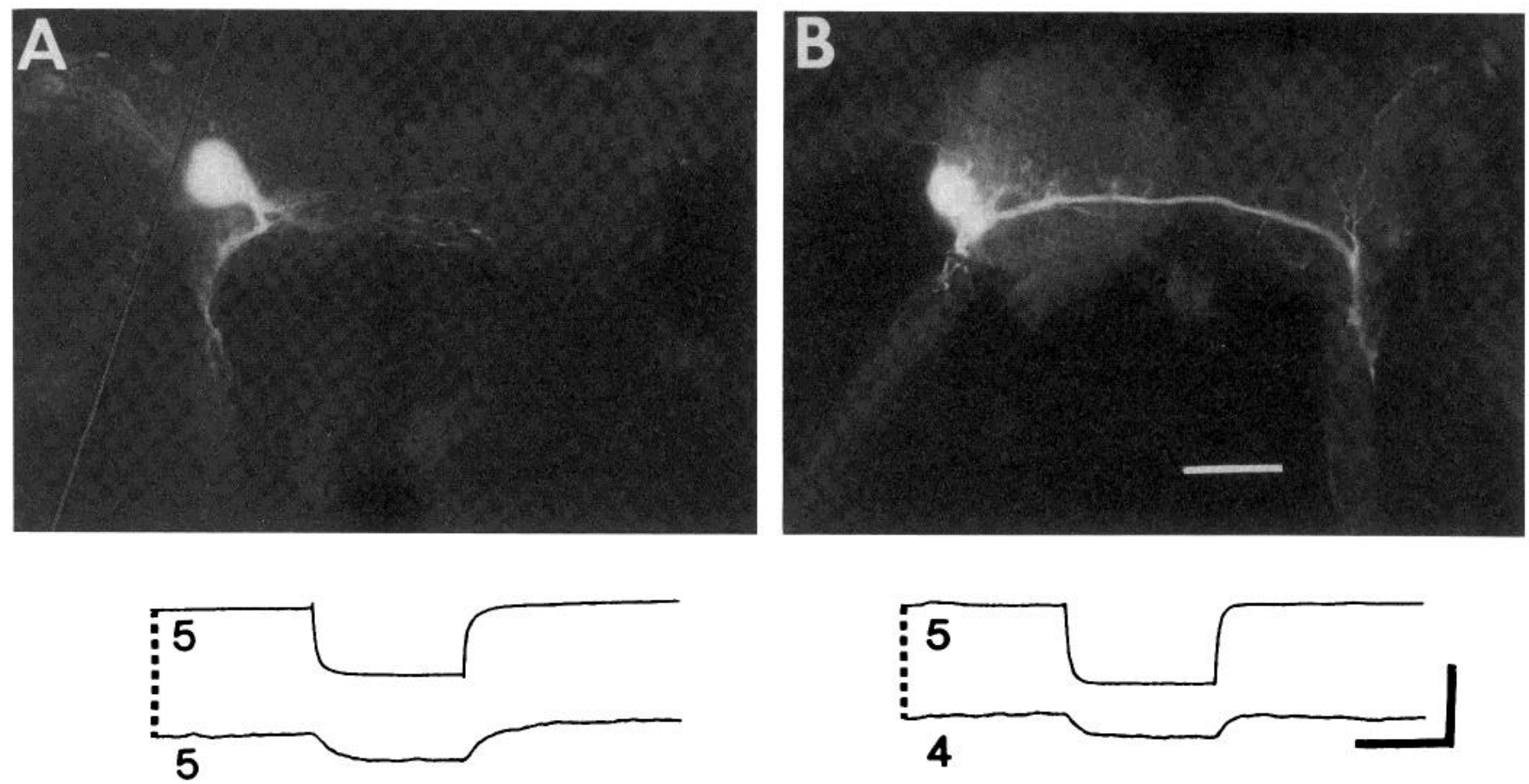

Figure 4. Morphology and connections between neuron 4 and neuron 5 after ET crush and culture. After axotomy by bilateral ET crushes, both neuron $5(A)$ and neuron $4(B)$ sprouted and produced large numbers of new central neuritic processes. These ganglia were cultured in Helisoma blood for 3 days before Lucifer Yellow injection. Electrical coupling measurements showed both 5-5 and 5-4 coupling in such preparations. Calibrations: 100- $\mu \mathrm{m}$ bar in photographs. Electrical records: top traces, $20 \mathrm{mV}$; lower traces, $5 \mathrm{mV} ; 2$ sec. crushed ETs, the axotomized neurons 5 and 4 both rapidly elaborated profuse neuronal sprouts in the central neuropile. This new morphological overlap provided a candidate substratum for forming new connections. Neuron 5 extended sprouts across the commissure and every preparation displayed 5-5 electrical coupling (Fig. 4A). In preparations with both neurons 5 filled, many inter-

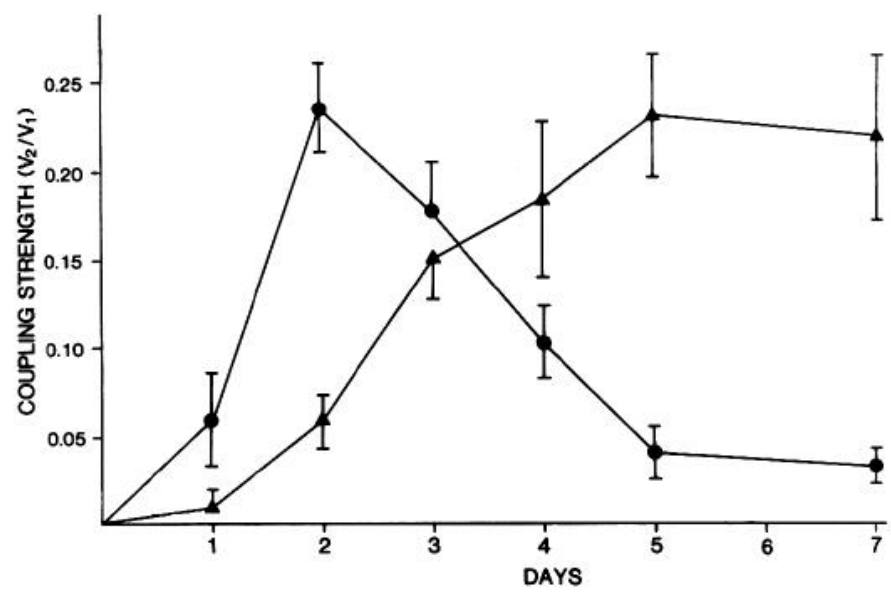

Figure 3. Time course of stable 5-5 and transient 5-4 electrical coupling. Ganglia with bilateral ET crushes were cultured in Helisoma blood for up to 7 days. Coupling between paired neurons 5 (triangles) and between neurons 5 and 4 (circles) was measured after varying times of culture. Neuron 5-4 coupling coefficients peaked at $0.24 \pm 0.03(n=11)$ on day 2 and fell thereafter. In contrast, $5-5$ coupling coefficients rose steadily to comparable levels and remained there. Values are mean coupling coefficients \pm SEM, $n=6$ to 13 for each point. 
twining neurites were evident (cf. Fig. 10B). Similarly neuron 4 also responded to axotomy by producing outgrowth in the central neuropile (Fig. $4 B$ ), which was overlapping with the growth from neuron 5 . Thus, central growth of both neurons was always correlated with the competence to make these novel connections.

Neurons not coupling to growing neurons 5 were found to lack central growth. Previous reports (Bulloch and Kater, 1981, 1982) showed that with ET crush, at least 25 identified buccal neurons (all with axons in the ETs) coupled to neuron 5 , but no connections were formed with the five pairs of protractor motoneurons (e.g., neuron 19). Because neuron 19 had no axon in the ETs, its central morphology did not change significantly in response to ET crush (Fig. $5 A$ ). In such ganglia, neuritic growth from neurons 4 and 5 clearly overlapped the normal axonal and dendritic projections of neurons 19 and would have seemed to allow direct contact, but essentially no electrical coupling ensued.

Recruitment of previously excluded neurons. We assessed 5-19 coupling capability when neuron 19 was experimentally induced to grow in the neuropile and asked:
(1) Would 5-19 connections form? (2) If neuron 19 were recruited to couple to neuron 5 , would the connections be stable or transient?

Crushing the commissure of buccal ganglia severed the commissural axon of neuron 19 and resulted in growth of central neurites (Fig. $5 B$ ). This growth by itself (i.e., without simultaneous ET crush) resulted in very little or no electrical coupling with the stable neuron 5 . Thus, in both complementary conditions ( 5 and not 19 growing or 19 and not 5 growing), 5-19 connections were lacking or extremely weak (coupling coefficient $<0.01$ ). On the other hand, when both commissure and ET were crushed, neurons 5 and 19 had concurrent central growth (Fig. 6), and 5-19 coupling was substantial (Fig. 7; mean $=0.17$ $\pm 0.016 ; n=9$ ). Thus induction of growing neurites which can interact with the growing neuron 5 alters the competence of those neurons to form novel connections.

A priori, novel 5-19 connections could have been transient, as were 5-4 connections, or stable, as were $5-5$ connections. A 7-day study of 5-19 connections showed that they were transient, with a peak at 2 days of culture (Fig. 8). The behavior of this newly recruited neuron thus
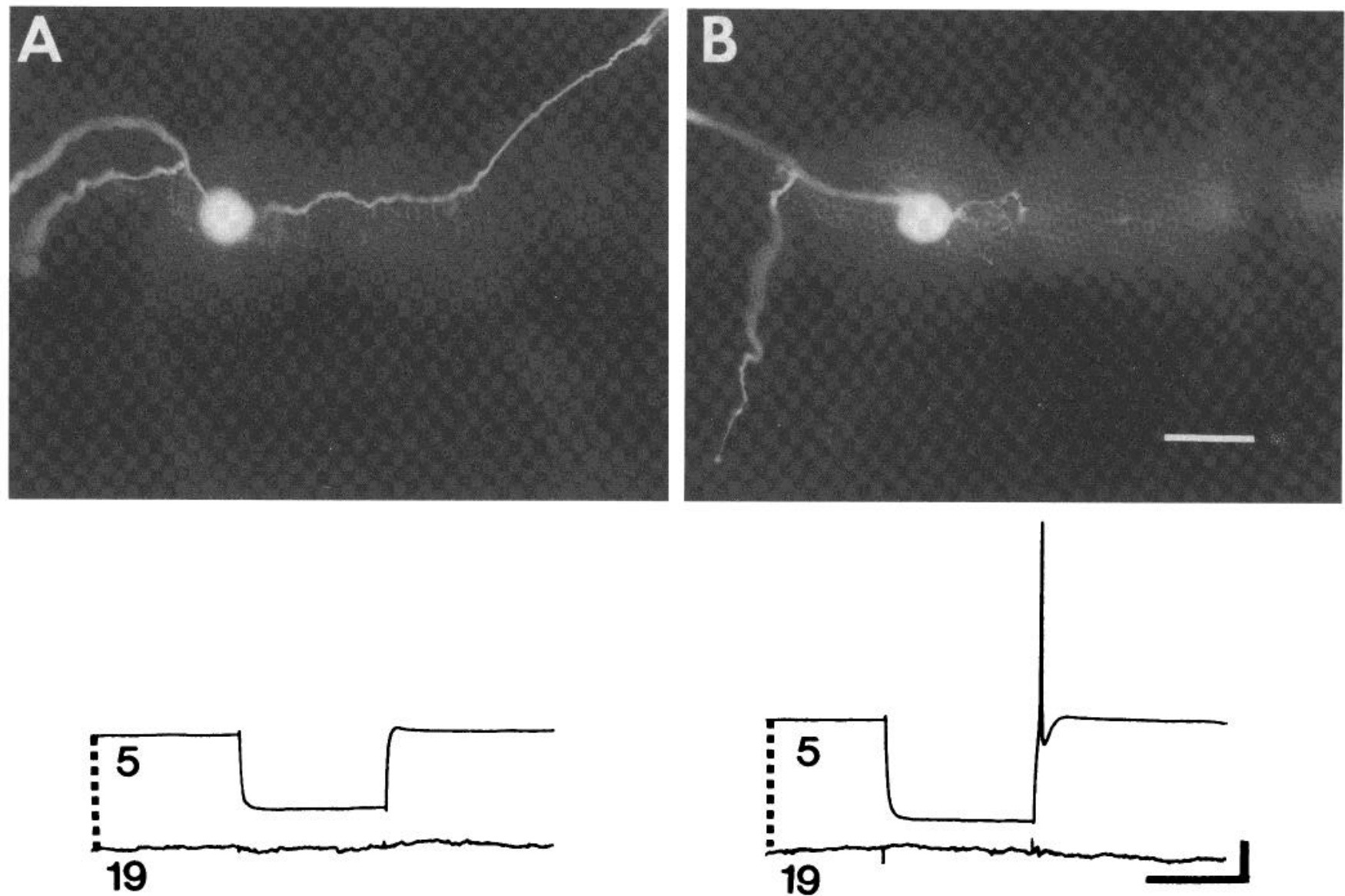

Figure 5. Responses of neuron 19 to ET $(A)$ or commissure $(B)$ crush. Neuron 19 produced no detectable central neurite growth in preparations with crushed ETs $(A, 3$-day culture). Neuron 5 in such preparations, however, produced extensive sprouting in the area of neuron 19 (cf., Fig. $4 A$ ). In preparations with crushed ETs $(A)$, very little or no 5-19 coupling was observed, as indicated by the voltage traces. Crushing the commissure but not the ETs $(B)$ resulted in elaboration of neuron 19 neurites in the central neuropile (3-day culture) but few or no central neurites from neuron 5 . Such preparations had very little or no 5-19 electrical coupling (voltage traces). Calibrations: 100- $\mu \mathrm{m}$ bar in photographs. Electrical records: top traces, $10 \mathrm{mV}$; lower traces, $1 \mathrm{mV}$; 2 sec. 

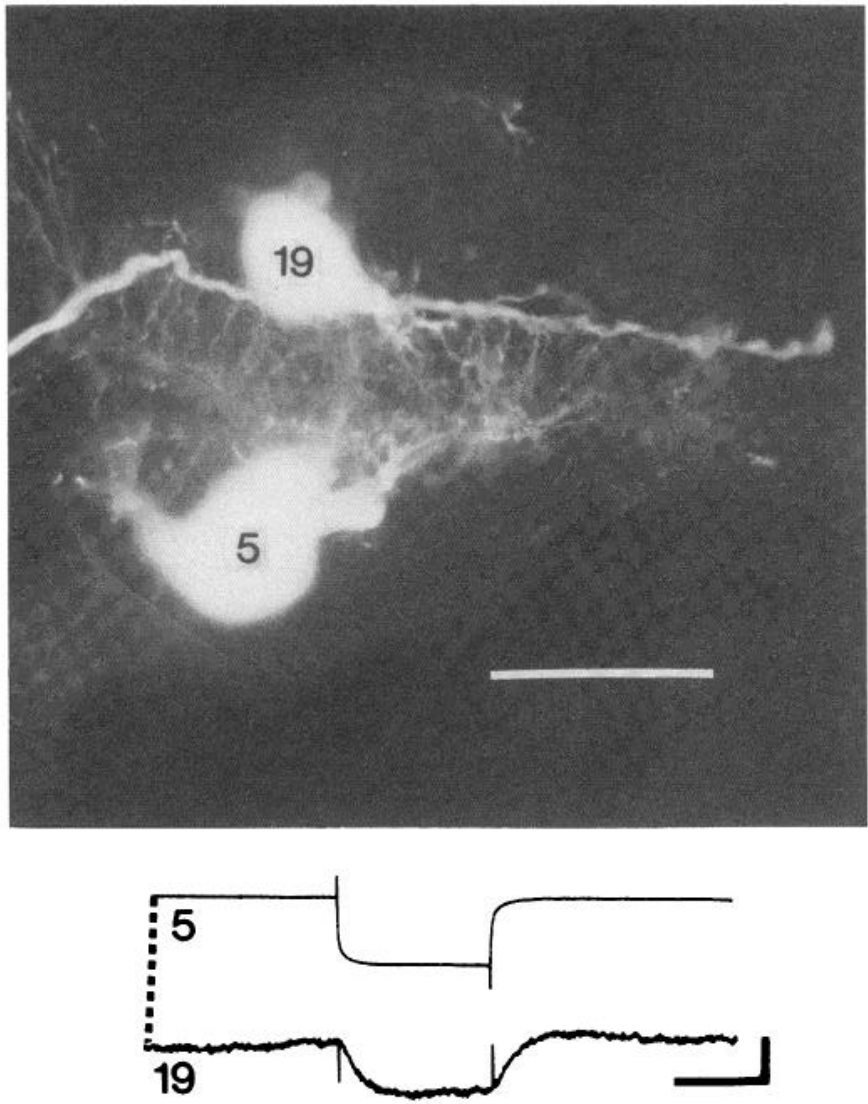

Figure 6. Neuron 5-19 coupling induced by crushing both ETs and commissure. Both neurons 19 and 5 were stimulated to grow centrally by crushing both ETs (neuron 5 axotomy) and the commissure (neuron 19 axotomy). In such preparations (3-day culture, only one side of the paired ganglia is shown), both neuron 5 and neuron 19 produced profuse, intermingling central outgrowth. This neurite outgrowth established the morphological substrate for novel electrotonic connections between neuron 5 and neuron 19 (voltage traces). Calibrations: $100-\mu \mathrm{m}$ bar in photograph. Electrical records: top trace, $10 \mathrm{mV}$; lower trace, $1 \mathrm{mV}$; 2 sec.

was essentially the same as that of other transiently connecting neurons.

The basis for even the very weak electrical coupling between neurons 5 and 19 when only one site was lesioned may be attributable to the presence of circuitous coupling pathways through numerous other neurons which also had their axons severed by nerve crush. For example, both neurons 4 and 19 have axons in the commissure. As expected, with only the commissure crushed, 19-4 connections were detected (although we made only a limited number of observations, $n=4)$. Under these conditions we also observed weak 5-4 connections (coupling coefficient $=0.03$ to 0.05 ; see also Bulloch and Kater, 1982). Thus current could flow indirectly from neuron 19 through neuron 4 and into neuron 5 . Such indirect pathways have not been studied in detail but serve to illustrate the limits imposed when making measurements within ganglia among many interacting neurons.

Exclusion from neuron 5 connection pattern. The above results show that an otherwise excluded neuron can be recruited to connect with neuron 5 by inducing

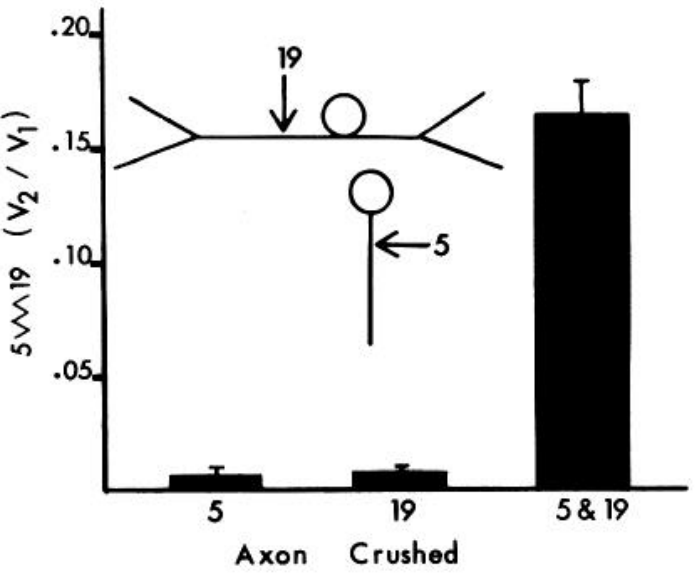

Figure 7. Summary of selectively induced growth and formation of novel 5-19 connections. When neuron 5 is axotomized by bilateral ET crush (5-arrow in schematic), profuse sprouts from neuron 5 fill the central neuropile, but only very low coupling is observed (three of eight preparations with detectable coupling). When neuron 19 is axotomized by commissure crush (19-arrow), central sprouting occurs, but coupling is again very low (six of seven with detectable coupling). When both neurons 5 and 19 grow centrally as a result of simultaneous ET and commissure crush, significantly much stronger 5-19 coupling occurs ( $p<0.001, t$ test). Values are mean \pm SEM, $n=7$ to 10 for each point, 2-day cultures.

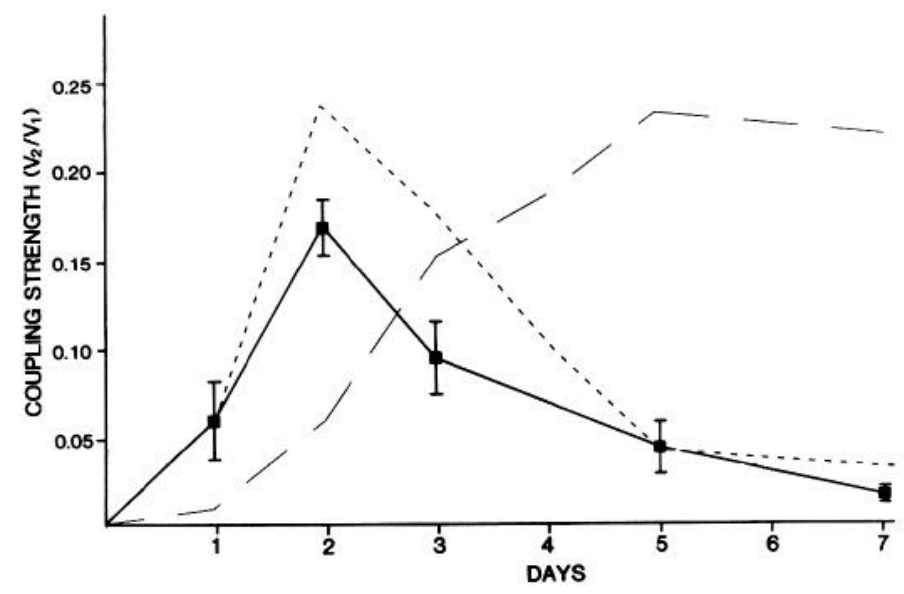

Figure 8. Time course of 5-19 electrical coupling with induced central growth of neurons 5 and 19. Assays of 5-19 coupling coefficients (squares) reveal that such coupling is transient over a 7-day time course. Replotted 5-4 (short dashes) and 5-5 (long dashes) coupling curves from Figure 3 are also represented for comparison. Points are mean \pm SEM, $n=6$ to 9 for each point.

central growth in both neurons. We then reassessed the ability of the "preferred" (i.e., selectively stabilized) 5 R$5 \mathrm{~L}$ connection to form when only one neuron (rather than both) was growing.

When neuron $5 \mathrm{R}$ was axotomized by unilateral right ET crush, 5R-5L electrical coupling after 5 days of culture (Fig. $9, A$ and $B$ ) was very weak (mean $=0.01 \pm 0.01$ ), and only four of seven preparations had any detectable coupling. In contrast, as noted above, $5 \mathrm{R}$ and $5 \mathrm{~L}$ of bilaterally crushed ET control preparations were all elec- 
trically coupled (Fig. $9 C$, mean $=0.20 \pm 0.03, n=7$ for this sample). Neurites of neuron $5 \mathrm{R}$ surrounded the more stable neuron 5L (Fig. 10) and thus the lack of 5R-5L coupling was apparently not based on lack of physical opportunity. The clear difference $(p<0.001)$ in coupling strength with individual versus conjoint growth lent further weight to the idea that the relative morphological stability of a neuron with an intact axon prevented its forming novel electrical connections during culture.

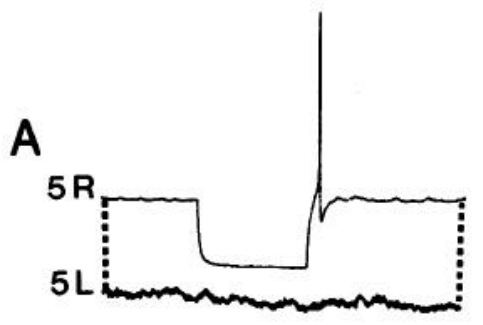

B

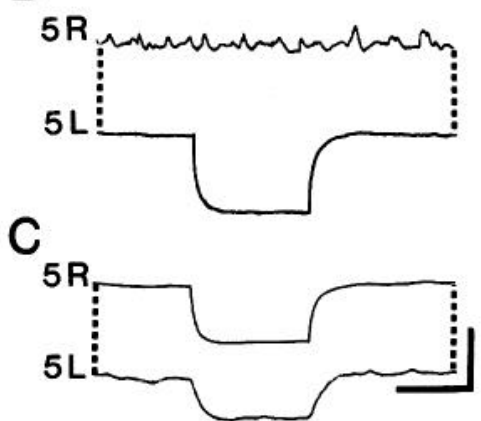

Figure 9. Lack of electrical coupling with unilateral right ET crush. Pairs of neurons 5 in unilaterally crushed ganglia with esophageal tissue remaining attached to the ETs lacked strong electrical coupling when assayed in either direction $(A$ and $B)$. Electrical coupling in a bilaterally crushed control preparation $(C)$ is shown at the same gain for comparison. Calibrations: In $A$ and $C$, upper traces, $20 \mathrm{mV}$; lower traces, $5 \mathrm{mV}$. Reversed gains in $B ; 2$ sec for all traces.

\section{Discussion}

Growth and the competence to form electrical synapses. Axotomy can be a potent signal, with effects ranging from biochemical changes (Grafstein, 1975) to changes in synaptic connectivity (Kuno and Llinás, 1970a, b; Matthews and Nelson, 1975; Bulloch et al., 1980). The most striking morphological response to axotomy is neurite outgrowth (e.g., Ramón y Cajal, 1928). In the present study, all neurons of the cultured buccal ganglia were axotomized when the ganglia were removed from the animal. This procedure induces sprouting, but it is restricted to the periphery, near the site of axotomy. Only proximally (i.e., near the cell body) axotomized neurons produce profuse central growth (Murphy and Kater, 1980; Bulloch and Kater, 1982; Hadley et al., 1982). This localization of growth as a function of axotomy position distinguishes between strict axotomy dependence versus localized growth dependence for enabling new electrical synapses to form. Even though neuron 19 is distally axotomized in crushed ET preparations, and thus is producing peripherally localized neurite outgrowth, strong 5-19 connections are not found. This was in spite of the ample overlap between growing sprouts of neuron 5 and the extant central projections of neuron 19. The same was true for neuron 5 -neuron 5 coupling (Figs. 9 and 10). Thus, physical proximity between growing regions of one neuron and stable, extant regions of a second neuron is not sufficient for formation of electrotonic synapses, even when both neurons have been axotomized.

The behavior of other growing regions of these neurons reinforces the concept of the relationship between growth and competence for making novel electrical connections. Growing peripheral axons of Helisoma neurons 5 also are capable of forming electrical connections with one another in peripheral nerve trunks when the ends of the two cut ETs are experimentally apposed in culture and the usual connection pathway is blocked (Hadley et al., 1982). Thus, simultaneous overlapping growth, and not
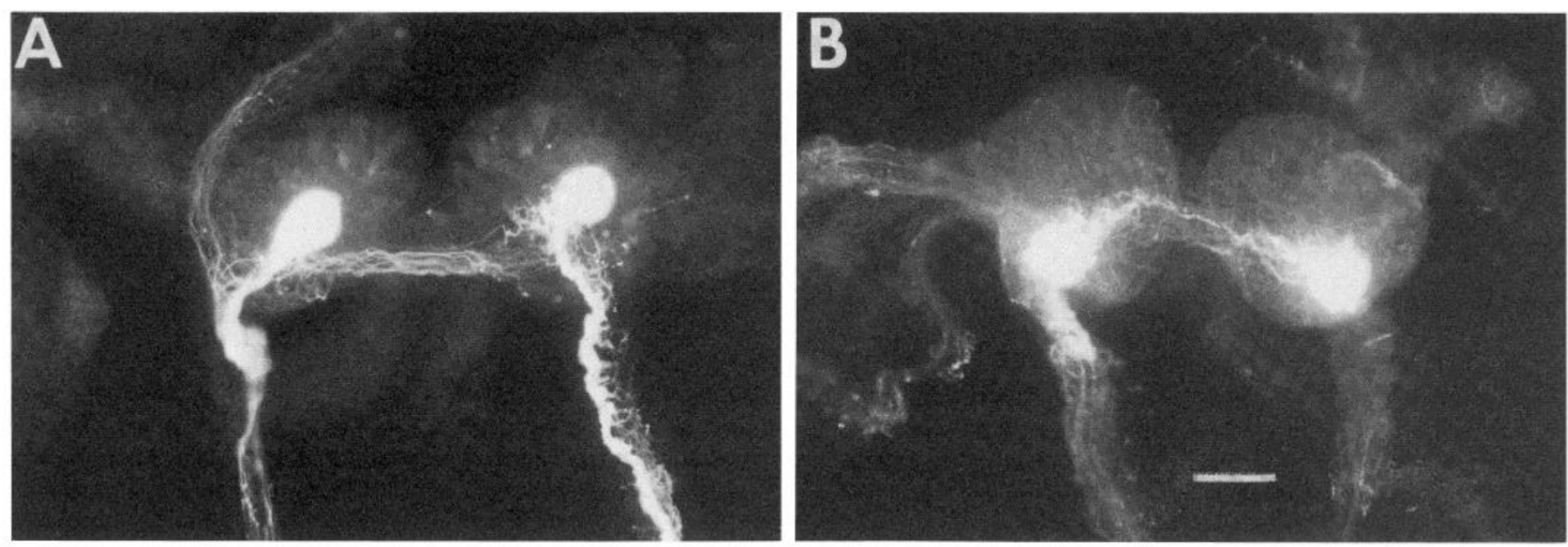

Figure 10. Neurite overlap with unilateral $(A)$ or bilateral $(B)$ crush of the ETs. Ganglia were cultured for 5 days with segments of esophagus remaining attached to the distal ends of the ETs to preserve the stability of uncrushed axons. Neuron 5R was axotomized by ET crush and its sprouts crossed the buccal ganglion, contacting the undamaged neuron $5 \mathrm{~L}(A)$. Bilaterally crushed control preparations $(B)$ showed similar amounts of sprouting in the buccal ganglion with strong electrical coupling (Fig. 9). $B a r$ in photograph, $100 \mu \mathrm{m}$. 
the specific part of the cell, is important for determining competence of neurons to connect. Additionally, these earlier data show that distally axotomized neurons (as in cultured, isolated ganglia) certainly are competent to make electrical connections, but their competence is localized to only the growing regions.

The formation of novel electrical connections is an intrinsic capability of individual neurons and is not dependent upon the systemic reorganization caused by nerve trunk damage. Evidence for this comes from our previous studies utilizing selective lesions of single axons within ganglia (Cohan et al., 1983) in which neurons undergo central growth in the relative absence of possible nerve crush influences. Such selectively axotomized neurons connect to other selectively axotomized neurons just as when the partners are axotomized by nerve crush. This provides support for the idea that central interactions among many axotomized neurons in a ganglionic environment are not required for electrical synapse formation. Thus, a state characterized by individual neuronal growth rather than a generalized organ-level response to axotomy (e.g., Rotshenker, 1978, 1979) can be isolated as a prerequisite for initial electrical coupling.

Operationally, it may be useful to consider that individual neurons that are capable of making connections are in a state different from those which are not competent. Each of these states is the reflection of multiple physiological processes, many of which may be unrecognized at present. Although the most striking manifestation of neurons competent to make electrical synapses is that of overlapping neurite outgrowth, the present experiments cannot unambiguously identify that growth is the only factor affecting electrical connections. Other, associated phenomena could play an important part. For example, in a stable morphological state, all available postsynaptic sites in the neuronal membrane could be physically occupied, and actual physical contact may not occur. New outgrowth may be required to produce new accessible space for connections to form. This kind of question is unresolvable in the ganglionic neuropile, but preliminary results of our experiments with isolated identified neurons in cell culture are consistent with a growth dependence hypothesis.

When pairs of single, identified neurons 5 are observed in cell culture (R. D. Hadley, S. B. Kater, and R. J. Bodnar, unpublished), contact between conjointly growing pairs results in electrical connections while direct physical contact between a growing and a nongrowing neuron results in no connection. Isolated neuronal cell culture also has shown that novel 5-19 (and other) connections can be direct (R. D. Hadley, C. S. Cohan, and S. B. Kater, unpublished data), with no intervening coupled cells as is possible in situ. All our results in cell culture are consistent with the idea that direct electrical connections occur only when there is direct interaction of actively growing regions of the neurons involved.

The connections described in this communication were assayed physiologically. The morphological substrates of these connections have not been pursued at the level of ultrastructure (i.e., gap junctions; Bennett and Goodenough, 1978). However, because large numbers of growing neurites crowding into the neuropile could reduce extra- cellular space, increasing extracellular resistance, it is conceivable that gap junctions could be unnecessary for physiological 5-4, 5-5, and 5-19 connections. This would make possible electrical coupling by current flow across nonspecialized cell membranes (e.g., Bennett and Trinkhaus, 1970). However, novel neuronal connections are indistinguishable from the genuine electrical synapses of normal buccal ganglia. Previous experiments (Bulloch and Kater, 1982) showed that novel 5-5 and 5-4 electrical connections are identical to extant 4-4 synapses in a number of electrophysiological characteristics, including a similar responsiveness to experimental reductions in $\mathrm{pH}$. All connections were reversibly decreased in strength in a similar fashion. It seems likely that novel connections and conventional buccal ganglion electrical synapses share a common substrate (i.e., low resistance intercellular pores) because of their similar characteristics.

Mechanisms for regulation of neuronal connections. In order for neurons to form an orderly and stable pattern of connections, some sort of "program" must be followed. One would like to describe the program as a set of rules governing the steps toward establishing circuitry. As a first approach in identifying such rules, Bulloch and Kater $(1981,1982)$ proposed a hierarchy of connection potentials for buccal ganglion neurons. Neuron 5 was found to couple to numerous other buccal neurons, but only 5-5 connections ("highest priority") remained stable and the others ("lower priority") were lost. The protractor motoneurons (e.g., neuron 19) were seen as the lowest priority group because they never coupled to neuron 5 . In the present communication we show that neuron 19 can be raised to the middle tier in this heirarchy, making transient connections to neuron 5 , when both cells are induced to grow centrally by simultaneous ET and commissure crushes. It seems then that the proposed lowest priority neurons are not unique in their inability to connect to neuron 5 , but that the basis for their exclusion in past experiments was their lack of central growth. Our working hypothesis that pairs of neurons must have overlapping areas of outgrowth, therefore, represents one possible "rule" for the initial formation of connections in this system.

It would be overly simplistic to state that simultaneous overlapping growth is the only factor in the formation of electrical connections. This phenomenon is only one aspect of multiple, interacting influences. The formation of neuronal connections is undoubtedly regulated at several levels and controlled by multiple mechanisms. It will not be surprising to find that indeed some neurons are excluded from the outset, no matter what their growth status may be.

Whereas early work focused on single mechanisms for the establishment of specific circuits (e.g., Sperry's (1963) hypothesis for retinotectal specificity), contemporary views recognize the likely interaction of several complementary mechanisms (cf. Fraser and Hunt, 1980). For example, as a first step in establishing specific connections, some factors must affect whether connections between given sets of neurons will ever occur. Only after the initial connections have formed can the phenomena of stabilization of appropriate synapses and elimination of inappropriate synapses further refine connectivity pat- 
terns (Changeux and Danchin, 1976). This occurs in development (e.g., Redfern, 1970; Purves and Lichtman, 1980) as well as regeneration of vertebrate (e.g., Cass et al., 1973; Brown and Ironton, 1978; Dennis and Yip, 1978) and invertebrate (Bulloch and Kater, 1981; Whitington, 1979) neurons. The remaining connections presumably must then be consolidated or stabilized (Lømo and Jansen, 1980). Such interactions of multiple mechanisms are likely to be general in the establishment of functioning neuronal circuits.

Summary. For any neuron there exists a set of other neurons with which it can connect under the appropriate conditions. This communciation represents an attempt at defining those "appropriate conditions." In Helisoma buccal ganglia, an adult neuron's new connections are determined by its growth status and the growth status of the neurons with which it interacts. This state dependence for formation of electrical synapses can be considered a "screening" mechanism, determining whether connections ever form. Such a mechanism predefines a group of neurons which are competent to connect to one another. Admittance to this group is possible once the condition of overlapping neurite outgrowth is met for a given pair of neurons. Selective mechanisms (i.e., when a neuron maintains some connections and breaks others) can operate only after initial, growth-dependent screening mechanisms have set up a matrix of connections from which selection can occur.

\section{References}

Bennett, M. V. L. (1966) Physiology of electrotonic junctions. Ann. N. Y. Acad. Sci. 137: 509-539.

Bennett, M. V. L., and D. A. Goodenough (1978) Gap junctions, electrotonic coupling, and intercellular communication. Neurosci. Res. Program Bull. 16: 373-486.

Bennett, M. V. L., and J. P. Trinkhaus (1970) Electrical coupling between embryonic cells by way of extracellular space and specialized junctions. J. Cell Biol. 44: 592-610.

Brown, M. C., and R. Ironton (1978) Sprouting and regression of neuromuscular synapses in partially denervated mammalian muscles. J. Physiol. (Lond.) 278: 325-348.

Bulloch, A. G. M., and S. B. Kater (1981) Selection of a novel connection by adult molluscan neurons. Science 212: 79-81.

Bulloch, A. G. M., and S. B. Kater (1982) Neurite outgrowth and selection of novel electrical connections by adult $\mathrm{Heli}$ soma neurons. J. Neurophysiol. 48: 569-583.

Bulloch, A. G. M., S. B. Kater, and A. D. Murphy (1980) Connectivity changes in an isolated molluscan ganglion during in vivo culture. J. Neurobiol. 11: 531-546.

Cass, D. T., T. J. Sutton, and R. F. Mark (1973) Competition between nerves for functional connections in axolotl muscles. Nature 243: 201-203.

Changeux, J. P., and A. Danchin (1976) Selective stabilisation of developing synapses as a mechanism for the specification of neuronal networks. Nature 214: 705-712.

Cohan, C. S., R. D. Hadley, and S. B. Kater (1983) "Zap axotomy": Localized fluorescent excitation of single dye-filled neurons induces growth by selective axotomy. Brain Res., in press.

Dennis, M. J., and J. W. Yip (1978) Formation and elimination of foreign synapses on adult salamander muscle. J. Physiol. (Lond.) 274: 299-310.

Dixon, W. J., and F. J. Massey, Jr. (1969) Introduction to
Statistical Analysis, Ed. 3, McGraw-Hill, New York.

Fraser, J. E., and R. K. Hunt (1980) Retinotectal specificity: Models and experiments in search of a mapping function. Annu. Rev. Neurosci. 3: 319-352.

Grafstein, B. (1975) The nerve cell body response to axotomy. Exp. Neurol. 48: 32-51.

Hadley, R. D., R. G. Wong, S. B. Kater, D. L. Barker, and A. G. M. Bulloch (1982) Formation of novel central and peripheral connections between molluscan central neurons in organ cultured ganglia. J. Neurobiol. 13: 217-230.

Kater, S. B., and C. R. S. Kaneko (1972) An endogenously bursting neuron in the gastropod mollusc, Helisoma trivolvis: Characterization of activity in vivo. J. Comp. Physiol. 79: 114.

Kater, S. B., and C. H. F. Rowell (1973) Integration of sensory and centrally programmed components in generation of cyclical feeding activity in Helisoma trivolvis. J. Neurophysiol. 36: 142-155.

Kuno, M., and R. Llinás (1970a) Enhancement of synaptic transmission by dendritic potentials in chromatolysed motoneurons of the cat. J. Physiol. (Lond.) 210: 807-821.

Kuno, M., and R. Llinás (1970b) Alterations of synaptic action in chromatolysed motoneurons of the cat. J. Physiol. (Lond.) 210: $823-838$.

Lømo, T., and J. K. S. Jansen (1980) Requirements for the formation and maintenance of neuromuscular connections. Curr. Top. Dev. Biol. 16: 253-281.

Matthews, M. R., and V. Nelson (1975) Development of structurally intact nerve endings from chromatolytic neurons of the rat superior cervical ganglion during depression of synaptic transmission induced by post ganglionic axotomy. J. Physiol. (Lond.) 245: 91-135

Murphy, A. D., and S. B. Kater (1978) Specific reinnervation of a target organ by a pair of identified molluscan neurons. Brain Res. 156: 322-328.

Murphy, A. D., and S. B. Kater (1980) Sprouting and functional regeneration of an identified neuron in Helisoma. Brain Res. 186: 251-272.

Murphy, A. D., R. D. Hadley, and S. B. Kater (1983) Axotomyinduced parallel increases in electrical and dye coupling between identified neurons of Helisoma. J. Neurosci., in press.

Purves, D., and J. W. Lichtman (1980) Elimination of synapses in the developing nervous system. Science 210: 153-157.

Ramón y Cajal, S. (1928) Degeneration and Regeneration of the Nervous System, R. M. May, transl., Hafner, New York, 1959.

Redfern, P. A. (1970) Neuromuscular transmission in newborn rats. J. Physiol. (Lond.) 209: 701-709.

Rotshenker, S. (1978) Sprouting of intact motor neurons induced by neuronal lesion in the absence of denervated muscle fibers and degenerating axons. Brain Res. 155: 354-356.

Rotshenker, S. (1979) Synapse formation in intact innervated cutaneous-pectoris muscles of the frog following denervation of the opposite muscle. J. Physiol. (Lond.) 292: 535-547.

Sperry, R. W. (1963) Chemoaffinity in the orderly growth of nerve fiber patterns and connections. Proc. Natl. Acad. Sci. U. S. A. 50: 703-710.

Stewart, W. W. (1978) Functional connections between cells as revealed by dye-coupling with a highly fluorescent naphthalamide tracer. Cell 14: 741-759.

Wong, R. G., R. D. Hadley, S. B. Kater, and G. C. Hauser (1981) Neurite outgrowth in molluscan organ and cell cultures: The role of conditioning factor(s). J. Neurosci. 1: 10081021

Whitington, P. M. (1979) The specificity of innervation of regenerating motor neurons in the cockroach. J. Comp. Neurol. 186: $465-472$. 